\title{
Atuação da equipe de enfermagem frente aos fatores de risco e prevenção de quedas
}

\section{na população idosa}

\author{
Nursing performance facing the risk factors and prevention of falls in the elderly population \\ Desempeño de enfermería frente a los factores de riesgo y prevención de caídas en la población \\ anciana
}

Recebido: 06/05/2021 | Revisado: 10/05/2021 | Aceito: 13/05/2021 | Publicado: 30/05/2021

\author{
Juliana Lima Magalhães \\ ORCID: https://orcid.org/0000-0001-7607-1196 \\ Instituto de Ensino Superior do Sul do Maranhão, Brasil \\ E-mail: julymagalhaes.lima@ hotmail.com \\ Aldenira Santos da Silva \\ ORCID: https://orcid.org/0000-0002-2805-812X \\ Instituto de Ensino Superior do Sul do Maranhão, Brasil \\ E-mail:niraenfer@hotmail.com
}

\begin{abstract}
Resumo
As quedas desempenham um papel importante na degradação da qualidade de vida dos idosos, suas consequências desenvolvem um impacto significativo na vida desses indivíduos, isso em razão da fragilidade física e fisiológica dos mesmos, gerando desafios pessoais envolvendo aspectos emocionais e no relacionamento familiar. Objetivo: Investigar a atuação da equipe de enfermagem quanto aos fatores de risco e a prevenção de quedas na população idosa. Metodologia: O estudo trata-se de uma revisão de integrativa com coleta de dados realizada mediante busca eletrônica, nas bases de dados National Library of Medicine (Pubmed), Scientifc Electronic Library Online (Scielo) e Literatura Latino-Americana e do Caribe em Ciências da Saúde (Lilacs), sobre a temática em questão, referente ao período de 2016 a 2021. Resultados: Foi observado que o espaço físico não adaptado é um dos principais fatores de risco para queda e que ele implica diretamente no aspecto econômico, outros fatores de ricos mencionados foram o desequilíbrio, tropeços, osteoporose e as medicações. Observou-se ainda medidas que a equipe de enfermagem pode estar realizando na prevenção das quedas, que foram: orientações, dinâmicas com a família e visitas domiciliares. Conclusão: No que se refere à análise dos dados presentes nesta revisão, é consenso entre os autores citados de que a enfermagem tem papel imprescindível na prevenção e cuidados referentes a quedas em idosos, além disso, atua diretamente para que os fatores de riscos sejam atenuados.
\end{abstract}

Palavras-chave: Fatores de risco; Idoso; Prevenção; Quedas.

\begin{abstract}
Falls play an important role in the degradation of the quality of life of the elderly, and their consequences have a significant impact on the lives of these individuals due to their physical and physiological fragility, generating personal challenges involving emotional aspects and family relationships. Objective: To investigate the nursing performance regarding risk factors and prevention of falls in the elderly population. Methodology: The study is an integrative review with data collection through an electronic search in the National Library of Medicine (Pubmed), Scientifc Electronic Library Online (Scielo), and Latin American and Caribbean Literature on Health Sciences (Lilacs) databases on the topic in question from 2016 to 2021. Results: It was observed that the non-adapted physical space is one of the main risk factors for falling and that it directly implies the economic aspect, other rich factors mentioned were imbalance, stumbling, osteoporosis and medications. We also observed measures that the nursing team can be taking to prevent falls, which were: orientations, dynamics with the family, and home visits. Conclusion: Regarding the analysis of the data present in this review, it is a consensus among the cited authors that nursing plays an essential role in the prevention and care of falls in the elderly, and that it acts directly to mitigate the risk factors.
\end{abstract}

Keywords: Nursing care; Elderly; Prevention; Falls.

\section{Resumen}

Las caídas juegan un papel importante en la degradación de la calidad de vida de las personas mayores, y sus consecuencias tienen un impacto significativo en la vida de estos individuos debido a su fragilidad física y fisiológica, generando desafíos personales que involucran aspectos emocionales y relaciones familiares. Objetivo: Investigar el desempeño de enfermería en cuanto a factores de riesgo y prevención de caídas en la población anciana. Metodología: El estudio es una revisión integradora con recolección de datos a través de una búsqueda electrónica en las bases de datos de la Biblioteca Nacional de Medicina (Pubmed), la Biblioteca Electrónica Científica en Línea (Scielo) y la 
Literatura Latinoamericana y del Caribe en Ciencias de la Salud (Lilacs) sobre el tema en pregunta de 2016 a 2021. Resultados: Se observó que el espacio físico no adaptado es uno de los principales factores de riesgo de caída y que implica directamente el aspecto económico, otros factores ricos mencionados fueron desequilibrio, tropiezos, osteoporosis y medicamentos. También observamos las medidas que puede estar tomando el equipo de enfermería para prevenir caídas, las cuales fueron: orientaciones, dinámicas con la familia y visitas domiciliarias. Conclusión: Respecto al análisis de los datos presentes en esta revisión, es un consenso entre los autores citados que la enfermería juega un papel fundamental en la prevención y atención de las caídas en el anciano, y que actúa directamente para mitigar los factores de riesgo.

Palabras clave: Factores de riesgo; Anciano; Prevención; Caídas.

\section{Introdução}

De acordo com a Organização Mundial da Saúde (OMS), idoso é todo indivíduo com 60 anos ou mais. O Brasil tem mais de 28 milhões de pessoas nessa faixa etária, número que representa $13 \%$ da população do país. E esse percentual tende a dobrar nas próximas décadas, segundo a Projeção da População, divulgada em 2018 pelo IBGE. Miranda (2016) enuncia que a transição demográfica inicia com a redução das taxas de morbimortalidade e, depois de um tempo, com a queda das taxas de natalidade, provocando significativas alterações na estrutura etária da população. Ademais, os avanços no campo da saúde e da tecnologia permitiram para a população com acesso a serviços públicos ou privados adequados, uma melhor qualidade de vida nessa fase (Tavares, et al., 2017).

Novas projeções populacionais são divulgadas anualmente de acordo com a Divisão de População da ONU esse levantamento é feito sobre todos os países e sobre o total mundial, e os resultados divulgados anteriormente não diferenciam dos mais recentes ambos indicam um crescimento da população idosa (De Oliveira, et al., 2020).

O processo de envelhecimento humano passa por um desgaste físico funcional do corpo onde ocorre a diminuição das respostas fisiológicas às ações do meio, causando danos a sua independência e autonomia que contribui para o surgimento de alterações psicológicas, de relacionamento pessoal, sexual e social (Miranda, et al., 2016). Diante do atual cenário é necessário medidas que favoreçam a promoção da inclusão e independência da pessoa idosa contribuindo diretamente na qualidade de vida e saúde desses indivíduos. Isso significa que para atender à demanda gerada por esse envelhecimento é preciso implantar mecanismos que fortaleçam o modelo de atenção à saúde do idoso, investindo inclusive na força de trabalho e na formação de profissionais que tenham habilidades para atuar na prevenção, no cuidado e na atenção integral à saúde da população idosa (Miranda, et al., 2016).

Todas as esferas presentes em uma sociedade são afetadas com o envelhecimento de sua população, há mudanças na economia, na saúde, na organização e também na política. No que se refere a saúde, as consequências desses acontecimentos se tornam presentes de forma mais imediata. Assim, urge a necessidade de se desenvolver novas técnicas e metodologias de atendimento específico para esses indivíduos, pois seus impactos crescem de acordo com o passar dos anos. Além disso, a utilização mais dos serviços e equipamentos de saúde passam a ser mais contínuos por parte da população em idades mais avançadas (Berlezi, et al., 2016).

Nessa continuidade, as quedas desempenham um papel importante na degradação da qualidade de vida dos idosos. As consequências são negativas no cotidiano das pessoas idosas, pois afeta de forma significativa a qualidade de vida e têm implicações para a família, pois em boa parte dos casos são os responsáveis pelos cuidados dessa população (Costa, et al., 2018).

A grande maioria das quedas ocorre no ambiente externo das residências, geralmente durante a realização de atividades cotidianas, como caminhar, mudar de posição ou ir ao banheiro, principalmente à noite, em que a maioria das lesões dentro de casa teria causas intrínsecas (Saad, 2016).

Nesse viés, os cuidados com os idosos podem ser prestados em diversos ambientes, podendo ser em domicílios, comunidades ou Instituições de Longa Permanência (ILPI). O cuidador tem características próprias que são externados mais 
em função do modelo presente na cultura do que por questões atreladas a planejamento. Dessa forma, desenvolver estratégias eficazes no fortalecimento da autonomia e independência para o envelhecimento saudável é de fundamental importância durante a utilização do espaço domiciliar e dos diversos serviços de saúde para os cuidados dos idosos (Rodrigues, et al., 2019).

Dessa maneira, é fundamental que haja conceitos que ajudem a entender o envelhecimento em sua totalidade. Apesar dos muitos conceitos disponíveis atualmente, é necessário entender seus significados e suas distinções. Embora tenham sentidos diferentes, todas estas perspectivas têm vindo a contribuir para contrariar uma visão negativa do envelhecimento humano, fazendo com que haja uma reflexão sobre a temática. Assim, há três tipos: envelhecimento bem-sucedido, envelhecimento produtivo e envelhecimento ativo (Gonçalves, 2015).

O idoso que vive no Brasil tem seu perfil epidemiológico diferente do que é apresentado por aqueles de faixas etárias mais jovens. Logo, ocorre com maior frequência a presença de apresentando doenças crônico-degenerativas como as osteoartroses, as insuficiências cardíaca e respiratória, o AVC (acidente vascular cerebral), a doença de Parkinson e também o Alzheimer (De Assis, et al., 2018).

Nesse seguimento, no que se refere a idosos que são atendidos em Unidades Básicas de Saúde, é notável que parcela significativa das quedas ocorram no ambiente domiciliar. Portanto, faz-se necessário programas de intervenção que suprimam os fatores de risco que estão associados à incapacidade funcional e, por consequência, o número de quedas. Além disso, o papel da enfermagem na prevenção é de fundamental importância, pois atua através da educação em saúde com palestras e orientações, conscientizando a população dos fatores e consequências relacionados a quedas em idosos (Santos, et al., 2020).

Nesse contexto, a pesquisa tem como objetivo investigar a atuação da equipe de enfermagem quanto aos fatores de risco e prevenção de quedas na população idosa.

\section{Metodologia}

Trata-se de uma pesquisa de revisão integrativa da literatura, de metodologia descritiva, apresentada de modo qualitativo. Configura-se como um tipo de revisão da literatura que reúne achados de estudos desenvolvidos mediante diferentes metodologias, permitindo aos revisores sintetizar resultados sem ferir a filiação epistemológica dos estudos empíricos incluídos (Soares, et al., 2014).

Portanto, um estudo integrativo configura-se como uma espécie de revisão de literatura, que pode coletar resultados de pesquisas desenvolvidas por diferentes métodos, possibilitando assim os revisores sintetizar os resultados, sem comprometer uma conexão epistemológica da pesquisa empírica contida. Para conduzir esse processo de maneira lógica, a revisão integrativa exige que os revisores, analisem e sintetizem os dados originais de maneira sistemática e rigorosa (Souza, et al., 2016).

O método qualitativo é considerado o trabalho mais adequado para a visão de pessoas e grupos, estando perfeitamente adaptado às características destinadas a responder a questões de personalidade e particularidade, pois é caracterizado pelas ciências sociais que não pode ser quantificado. Portanto, esse método atua sobre o universo de causas, crenças, significados, atitudes, ideais e valores que produzem respostas importantes, explicativas e profundas que têm a capacidade de gerar novos relatos não adequados para dados quantitativos (Souza, et al., 2016).

A coleta de dados foi realizada mediante busca eletrônica, nas bases de dados National Library of Medicine (PUBMED), Scientific Electronic Library Online (SCIELO) e Literatura Latino-Americana e do Caribe em Ciências da Saúde (LILACS). A temática em questão, foi desenvolvida no período de 2015 a 2020, tendo o enfoque de pesquisa voltado para a questão norteadora que ''Qual a importância da atuação da equipe de enfermagem frente aos fatores de risco e prevenção de 
quedas na população idosa?'”. Para compor a buscas dos dados, foram utilizados os Descritores de Ciências da Saúde (DeCS): Fatores de risco. Idoso. Prevenção. Quedas. Os operadores booleanos OR e AND também são usados junto aos descritores.

Para os critérios de inclusão foram utilizados artigos completos em língua portuguesa, disponíveis eletronicamente e que obedeçam à temática supracitada. Referente aos critérios de exclusão estão: relatos de experiência, estudos de revisão, livros e pesquisas duplicadas, monografias e dissertações.

Assim, para análise de conteúdo e classificação dos artigos foram seguidos os seguintes passos, segundo Bardin (1997):

a) Pré-análise: leitura flutuante do material coletado; constituição do corpus da pesquisa;

b) Exploração do material: recorte em unidades de registro de contexto; codificação e classificação segundo categorias empíricas e teóricas;

c) Tratamento dos dados e interpretação: análise final dos dados obtidos. Não sendo necessária a aprovação por um Comitê de Ética em Pesquisa por se tratar de uma pesquisa bibliográfica.

Mediante leitura e análise dos estudos, foi possível se estabelecer um conjunto de pesquisas sobre a temática. Foi então, desenvolvido uma tabela para apresentação dos artigos, os mesmos receberão um codinome de letra e número E1, E2, E3... E7.

\section{Resultados e Discussão}

O Quadro 1 a seguir, apresenta o compilados dos resultados obtidos, que foram incluídos no artigo, sendo possível uma visualização completa e organizada das pesquisas.

Quadro 1. Artigos que tratam sobre prevenção de quedas em idosos e os cuidados da enfermagem.

\begin{tabular}{|c|c|c|c|c|}
\hline \multicolumn{2}{|c|}{ AUTOR/ANO } & \multirow{2}{*}{$\begin{array}{c}\text { REVISTA } \\
\text { /BASE DE } \\
\text { DADOS } \\
\text { Acta Paulista de } \\
\text { Enfermagem } \\
\text { (SCIELO) }\end{array}$} & \multirow{2}{*}{$\begin{array}{l}\text { METODOLOGIA } \\
\text { Estudo } \\
\text { transversal }\end{array}$} & \multirow{2}{*}{\begin{tabular}{|c|} 
RESULTADOS \\
Foi observado que, os aspectos preventivos mais eficazes para a \\
queda em idosos se apresentou na adaptação fisíca do recinto em \\
que o idoso está inserido. Alguns dos mencionados foram: \\
apoios de parede, manter espaços abertos (retirada de tapetes, \\
caixas, mesas de centro, entre outras), pisos da casa secos, anti- \\
derrapantes e foscos. Os fatores de risco mais frequentes, \\
quedas, o escorregar, tropeçar, por causa do calçado, outros \\
motivos e falta de adaptação em casa.
\end{tabular}} \\
\hline E1 & $\begin{array}{l}\text { Giacomini, et al., } \\
\text { (2020) }\end{array}$ & & & \\
\hline E2 & $\begin{array}{l}\text { Teixeira } \\
(2020)\end{array}$ & $\begin{array}{c}\text { Instituto } \\
\text { Politécnico de } \\
\text { Bragança } \\
\text { (SCIELO) }\end{array}$ & Estudo transversal & $\begin{array}{c}\text { Os resultados obtidos sugerem que, após os dois meses de } \\
\text { intervenção, o grupo teve melhor desempenho na realização de } \\
\text { todas as atividades da Escala de Equilíbrio de Berg, indicando } \\
\text { melhora estatisticamente significativa no equilíbrio corporal e na } \\
\text { estimativa do risco de quedas. }\end{array}$ \\
\hline E3 & $\begin{array}{l}\text { Silva \& Bolpato } \\
\text { (2017) }\end{array}$ & $\begin{array}{l}\text { Jornal Health } \\
\text { SPEPS } \\
\text { (SCIELO) }\end{array}$ & Estudo descritivo & $\begin{array}{c}\text { Observou-se que os enfermeiros possuem um papel fundamental } \\
\text { na educação de saúde dos idosos, a pesquisa apresentou que as } \\
\text { medidas preventivas como as palestras participativas entre } \\
\text { idoso/familia, facilita o entendimento para um trabalho em } \\
\text { conjunto, explicar para o idoso o que pode acontecer caso o } \\
\text { mesmo não pratique o auto cuidado, e para familia, incentivar o } \\
\text { idoso a ter maior autonomia. }\end{array}$ \\
\hline
\end{tabular}




\begin{tabular}{|c|c|c|c|c|}
\hline $\mathbf{E 4}$ & $\begin{array}{l}\text { De Freitas, et al., } \\
\text { (2018) }\end{array}$ & $\begin{array}{l}\text { Revista da Escola } \\
\text { de Enfermagem } \\
\text { (LILACS) }\end{array}$ & $\begin{array}{l}\text { Estudo longitudinal } \\
\text { retrospectivo }\end{array}$ & $\begin{array}{l}\text { Dessa maneira, a incidência de quedas acompanhou a } \\
\text { implementação de ações preventivas, sugerindo que o impacto } \\
\text { dessas intervenções na redução da ocorrência do evento. } \\
\text { Pricipais fatores de riscos observados foram, pisos } \\
\text { escorregadios, desequilibrios, quedas e espaço fisico não } \\
\text { adaptado. }\end{array}$ \\
\hline $\mathbf{E 5}$ & $\begin{array}{c}\text { De Souza \& } \\
\text { Oliveira } \\
(2019)\end{array}$ & $\begin{array}{l}\text { Revista Baiana de } \\
\text { Enfermagem } \\
\text { (LILACS) }\end{array}$ & $\begin{array}{l}\text { Estudo transversal e } \\
\text { descritivo }\end{array}$ & $\begin{array}{c}\text { Os principais fatores encontrados neste estudo foram idade } \\
\text { superior a } 80 \text { anos, existência de comorbidades como Alzheimer } \\
\text { e diabetes, uso de auxílio para deambulação, pisos } \\
\text { escorregadios, histórico de quedas e marcha com } \\
\text { comprometimento específico, além de histórico de osteoporose e } \\
\text { uso de medicações. }\end{array}$ \\
\hline E6 & $\begin{array}{c}\text { Costa, et al., } \\
\text { (2017) }\end{array}$ & $\begin{array}{l}\text { Revista Saúde } \\
\text { (PUBMED) }\end{array}$ & Estudo transversal & $\begin{array}{l}\text { Os resultados sugerem que, a ocorrência de trauma por queda } \\
\text { em idosos está relacionada a maior fragilidade óssea decorrente } \\
\text { da osteoporose que estes indivíduos costumam apresentar. Estas } \\
\text { quedas ocorrem geralmente no ambiente doméstico e tem vários } \\
\text { fatores associados como: distúrbios neurológicos, uso de } \\
\text { medicamentos que atuam sobre os psiquismos que podem } \\
\text { atingir o equilíbrio, tempo de reação, utilização de calçados } \\
\text { inadequados, disposição dos móveis, utilização de tapetes nos } \\
\text { próprios domicílios e diversas patologias. }\end{array}$ \\
\hline E7 & $\begin{array}{l}\text { Alves, et al., } \\
\text { (2017) }\end{array}$ & $\begin{array}{c}\text { Revista Brasileira } \\
\text { de Geriatria } \\
\text { (SCIELO) }\end{array}$ & $\begin{array}{l}\text { Estudo de corte } \\
\text { transversal }\end{array}$ & $\begin{array}{l}\text { Houve } 36,41 \% \text { de quedas em idosos, sendo } 45,95 \% \text { ocorrendo } \\
\text { fora de casa e } 39,78 \% \text { usavam medicamentos, } 87,71 \text { sofreram } \\
\text { AVC. Dos idosos que caíram e sofreu fratura }(18,67 \%), 50 \% \text { já } \\
\text { tiveram acidente vascular cerebral e } 50 \% \text { doença renal crônica, } \\
61,54 \% \text { interromperam suas atividades diárias após a queda. } \\
\text { Sendo assim, os fatores mais intimamente relacionados foram o } \\
\text { uso de drogas, vítimas de derrame e } 61,54 \% \text { daqueles com } \\
\text { fratura interromperam suas atividades diárias. }\end{array}$ \\
\hline
\end{tabular}

Fonte: Autores (2021).

Mediante ao quadro exposto, em ordem cronológica, foi possível se observar que os artigos referentes ao ano de 2017 foram 3, tendo por metodologia, 2 de estudos transversais e um estudo descritivo, no ano de 2018, obteve-se 1 estudo sendo ele de característica longitudinal retrospectiva, apenas 1 pesquisa para o ano de 2019, sendo ela de metodologia transversal descritiva, e os mais atuais ficaram para ano de 2020, onde obteve-se 2 trabalhos, onde ambos apresentaram-se por ser de aspecto metodológico transversal.

Observou-se nos estudos de E1, E4, E5, E6 e E7, que os fatores de risco são uma grande problemática, e que os mais comuns entre os estudos se apresentaram como, espaço fisíco não adaptado, tropeços, e desequilíbrios, isso devido à fragilidade dos idosos a queda, que se torna um agravante em sua saúde e pode gerar graves consequências. Vale ressaltar que, o espaço fisico não adaptado entrou como a mais frequente entre todos os fatores de risco, abre-se assim um questionamento, será se a dificuldade de se ter espaços adaptados para a população idosa, esta associado ao aspecto econômico?

Em estudo sobre o diagnóstico de enfermagem e os fatores de risco sobre as quedas em idosos na atenção primária, Sousa, et al., (2016) afirmam que o risco de quedas em idosos acontece, principalmente em casos de doenças crônicas, uso de dispositivos auxiliares, dificuldades na marcha, alteração na função cognitiva e anemia. Em relação ao fator extrinseco que aumentou o risco de quedas nos idosos, o uso de tapetes soltos é destacado pelos autores. Afirmam ainda que, a adaptação dos espaços fisicos para os idosos é um desafio para as familias, pois, é explicado que os materias para se adaptar 
são caros, dentre esses estão, pisos focos e antiderrapantes, apoios de parede, camas e colchões, cadeiras especiais, entre outros.

Em relação as condições que levam a queda, os fatores de risco que geram essa consequência, e de modo repetido são tropeços e perda de equilíbrio. A maioria das quedas é causado pela perda do equilíbrio dinâmico, ou seja, ocorrem durante a locomoção, principalmente por tropeços e escorregões durante a marcha. Pesquisas indicam que o envelhecimento e a mudança no padrão de caminhar e o equilíbrio como a disfunção da marcha é o problema mais comum na população idosa, sua prevalência aumenta com a idade (Moraes, et al., 2017).

Souza, et al., (2016) citam as principais intervenções como metodos preventivos, que devem ser introduzidas na residência do idoso para que quedas e lesões sejam evitadas atravéz da enfermagem que atua com visitas domiciliares preventidas, dinânamica com a familia e idoso, aconselhamentos sobre modificações domiciliares, reforço comportamental e modificações de pavimentos, logo, essas ações foram preponderantes entre os achados apresentados.

Nesse sentido Souza, et al., (2017) relatam que a atuação da enfermagem para idosos vulneráveis à quedas, volta-se principalmente à atividades de educação em saúde, de forma coletiva com a família, a fim de incluir todos no cuidado e atenção aos riscos das atividades do cotidiano. Para tanto, outras intervenções devem ser propostas mediante a identificação de fragilidades familiares ou estado de saúde do idoso, isso cabe, na realização de visitas domiciliares, onde a equipe irá identificar, os fatores de risco presentes e aplicar medidas de prevenção, tais como: adaptação do espaço fisico, orientações e dinâmicas entre familiar e idoso.

As Diretrizes de Habitação Segura ajudarão a prevenir quedas e, assim, evitar a ocorrência de fraturas. Ao enfatizar um ambiente seguro, trabalharemos com o meio ambiente ao mesmo tempo, pois o conceito de meio ambiente segue principalmente três eixos: a) Um espaço confortável privilegia a privacidade e a personalidade do sujeito, e preza pelos elementos ambientais que interagir com as pessoas, como cor, cheiro, som, luz, forma, etc., e garantir o conforto dos trabalhadores e usuários; b) Por meio da ação e reflexão do processo de trabalho, permissão a criação de um espaço subjetivo e temático de conferência; c) Utilizar como ferramenta facilitadora do processo de trabalho o espaço é propício à otimização de recursos, humanização, entusiasmo e serviço decidido (Freitas, et al., 2019).

Nos estudos de E2 e E3 os autores permitiram identificar e analisar a incidência de quedas em ambiente hospitalar, bem como suas relações com as ações implementadas pela equipe de enfermagem ao longo de 5 anos, mostrando a importância das intervenções preventivas na redução da ocorrência de quedas. No panorama atual da qualidade e segurança da saúde, apresentar resultados de ações preventivas para um dos principais incidentes de segurança, como quedas, é fundamental para a prática de enfermagem e saúde, podendo auxiliar as instituições hospitalares no desenvolvimento de intervenções, metas e na gestão dos incidentes.

A equipe de enfermagem deve direcionar suas ações para a educação em saúde, com ênfase no autocuidado, além de avaliar o perfil do idoso, para facilitar a identificação dos fatores de risco, realizar anamnese e exame físico visando à redução de agravos. Além disso, é considerado essencial estimular mudanças nos hábitos de vida nocivos, como o sedentarismo e o uso irracional de medicamentos. Portanto, é importante que o enfermeiro e a equipe de enfermagem iniciem e fortaleçam o estabelecimento de ações/estratégias que visem prevenir a queda de pessoas idosas, seja em hospital, lar de idosos ou residência. Por fim, proceder a uma avaliação criteriosa do local de residência do idoso e incentivar a reestruturação das residências com base nas necessidades detectadas, com o objetivo de minimizar os riscos, bem como identificar o estado de família do idoso e o apoio social (Freitas, et al., 2019).

Nos resultados de E1, E2, E4 e E5, é sugerido que a ocorrência de trauma por quedas em idosos está ligada à maior fragilidade óssea resultante da osteoporose que essas pessoas geralmente apresentam. Estas quedas geralmente ocorrem no ambiente doméstico e têm vários fatores associados e predisponentes, como: distúrbios neurológicos, uso de medicamentos que 
atuam a médiuns que podem alcançar equilíbrio, déficit muscular, força, tempo de reação e flexibilidade, uso de sapatos inadequados, móveis, usando tapetes em domicílios e várias patologias.

De acordo com Moraes, et al., (2017) é ressaltado que na literatura, existem alguns estudos que mostram que $75 \%$ dos idosos caem dentro de casa em comparação com aqueles que caem fora de sua residência. O ambiente doméstico é o mais propenso a causar acidentes por quedas no quarto $(25,2 \%)$, seguido da cozinha $(16,8 \%)$ e do banheiro $(14,5 \%)$. Isso pode estar relacionado devido as pessoas idosas passam muito tempo em ambiente domiciliar, por se tratar de um lugar, mais seguro e familiar. Em casa, os idosos podem perder a atenção porque estão mais confiantes e familiarizados com os espaços do recinto.

Vale ressaltar que em E5, a osteoporose e o uso de medicamentos foram citados, como fatores de risco para a queda em idosos, visto a osteoporose uma patologia que causa maior vulnerabilidade e fragilidade e as medicações posuirem reações adversas para esse público. É necessário que os enfermeiros saibam como determinar os fatores de risco para a osteoporose: mulheres em menopausa anterior 45 anos; com múltiplas gestações em seu histórico; consumiu pouco cálcio ao longo da vida; falta de atividade física; peso corporal abaixo do normal; pele branca, história familiar de osteoporose e fumantes. Esse conhecimento proporcionará ao enfermeiro maior condição de fornecer orientações suficientes para prevenir essa importante condição patológica que em por consequência às quedas (Freitas, et al., 2019).

Os medicamentos de origem crônica podem aumentar o risco de quedas e causar sintomas como hipotensão ortostática, disfunção cognitiva, distúrbios do equilíbrio, tontura, tontura, sonolência, disfunção motora, reflexos enfraquecidos e mudanças visuais. Portanto, o uso de medicamentos na população idosa, possui múltiplos fatores que podem levar à consequências indesejáveis (Beijo, et al., 2017).

Essa correlação é explicada por Moraes, et al., (2017) onde pode ser entendida por meio do comprometimento dos reflexos que se desenvolvem devido aos efeitos adversos dos medicamentos. Os reflexos diminuem por conta do processo natural de envelhecimento, sendo potencializados com o uso de medicamentos, dificultando a mobilidade e funcionabilidade do corpo, consequentemente ocasionando quedas. Dessa forma, os profissionais da área de gerontologica podem identificar os possíveis contribuintes para a queda. Vale ressaltar que sugerido como cuidado o ato de se investigar o histórico das quedas e explorar os resultados proveniente da mesma, se tropeçou, se houve perda de consciência, tontura, se houve alterações nas medicações como: vasodilatadores, sedativos e diuréticos, e por fim questionar quanto a visão, se houve uso de álcool ou fumo.

\section{Conclusão}

No que se refere à análise dos dados presentes nesta revisão de literatura, é consenso entre os autores citados de que a enfermagem tem papel imprescindível na prevenção e cuidados referentes a quedas em idosos, além disso, atua diretamente para que os fatores de riscos sejam atenuados. Vale ressaltar que, foi observado nos estudos, a questão econômica como desafio frente as quedas, visto que a adaptação de uma residência, gera custos para o idoso e as famílias, sendo assim uma questão não apenas de saúde, mas econômica e social.

Assim, o papel da enfermagem está relacionado principalmente às atividades de educação em saúde, coletivamente com a família, para incluir todos os cuidados e atenção aos riscos das atividades diárias. Para isso, outras intervenções devem ser propostas identificando a fragilidade da família ou o estado de saúde dos idosos. Vale ressaltar que quando o idoso sore com a queda, há uma tendência na redução de suas atividades diárias, seja pelo medo de correr o risco de cair novamente, ou seja pela atitude protetora de familiares e cuidadores. Essa redução pode levar à imobilidade, falta de produtividade, problemas emocionais e subsequente atrofia muscular, facilitando a recorrência de quedas.

Concluiu-se que o histórico de quedas mencionado nos estudos, e a não adaptação dos recintos onde os idosos se encontram, afetam significativamente na incidência de quedas, indicando a importância da atuação de enfermagem para o 
enfrentamento desses fatores por meio de programas de atenção à saúde, que tenham por intuito a orientação e a aplicabilidade de situações dinâmicas que envolvam familiar e idoso.

Sugere-se para que trabalhos futuros abordem o tema de forma prática e também de forma social, englobando a comunidade de forma educativa para que diversas famílias sejam atendidas. Assim, o tema conseguirá atingir o público que realmente importa, que são os idosos. Estratégias de intervenção nas residências também se faz importantes, buscando, através de educação em saúde uma melhor forma de adaptação das residências para que as quedas sejam diminuídas.

\section{Referências}

Avanecean, D., Calliste, D., Contreras, T., Lim, Y., \& Fitzpatrick, A. (2017). Eficácia das intervenções centradas no paciente em quedas no ambiente de cuidados intensivos em comparação com o tratamento usual: uma revisão sistemática. JBI Evidence Synthesis, 15 (12), $3006-3048$.

Beijo, L. A., Avelar, F. G., da Costa Teodoro, L., Goyatá, S. L. T., \& ARAÚJO, M. P. (2017). Fatores relacionados à ocorrência de queda de idosos. Revista da Universidade Vale do Rio Verde, 15(1), 38-48.

Berlezi, E. M., Farias, A. M., Dallazen, F., Oliveira, K. R., Pillatt, A. P., \& Fortes, C. K. (2016). Como está a capacidade funcional de idosos residentes em comunidades com taxa de envelhecimento populacional acelerado? Revista Brasileira de Geriatria e Gerontologia, 19(4), 643-652.

Costa, J. C., De Lima, M. O., Pequeno, J. A., de Lima, M. O., Lima, T. O., \& de Lima Junior, A. A. (2018). Trauma por queda em idosos e a assistência de enfermagem. Revista Saúde-UNG-Ser, 11(1 ESP), 40.

de Freitas Luzia, M., Cassola, T. P., Suzuki, L. M., Dias, V. L. M., de Pinho, L. B., \& de Fátima Lucena, A. (2018). Incidence of falls and preventive actions in a University Hospital. Rev Esc Enferm USP, 52, e03308.

de Moura Sá, G. G., de Assis Brito, M., do Vale Carvalho, N., dos Santos, A. M. R., da Rocha, S. S., \& da Silva, G. R. F. (2018, December). Enfermagem e tecnologias para prevenção de queda em idosos: revisão integrativa. In Anais do I Congresso Norte Nordeste de Tecnologias em Saúde (Vol. 1, No. 1).

de Oliveira Reis, A., Martins, S., \& Pinto, T. R. G. S. (2020). Estratégia Brasil Amigo da Pessoa Idosa em governos locais brasileiros. GIGAPP Estudios Working Papers, 7(150-165), 411-428.

de Souza Pedro, L., \& de Oliveira Faria, J. (2019). Desafios da prevenção de quedas em idosos na atenção primária à saúde. Revista Baiana de Enfermagem33,.

dos Santos, B. W., \& Baixinho, C. L. (2019). Intervenções de enfermagem no espaço físico da casa para prevenir a queda no idoso: Revisão Integrativa da Literatura. CIAIQ2019, 2, 91-100.

Giacomini, S. B. L., Fhon, J. R., \& Rodrigues, R. A. P. (2020). Fragilidade e risco de queda em idosos que vivem no domicílio. Acta Paulista de Enfermagem, 33 .

Gonçalves, C. D. (2015). Envelhecimento bem-sucedido, envelhecimento produtivo e envelhecimento ativo: reflexões. Estudos Interdisciplinares sobre o Envelhecimento, 20(2).

Miranda, G. M. D., Mendes, A. D. C. G., \& Da Silva, A. L. A. (2016). O envelhecimento populacional brasileiro: desafios e consequências sociais atuais e futuras. Revista Brasileira de Geriatria e Gerontologia, 19(3), 507-519.

Rodrigues, R. A. P., Bueno, A. D. A., Casemiro, F. G., Cunha, A. N. D., Carvalho, L. P. N. D., Almeida, V. C., \& Seredynskyj, F. L. (2019). Supuestos de buenas prácticas en la atención domiciliaria de las personas mayores: una revisión sistemática. Revista Brasileira de Enfermagem, $72,302-310$.

Saad, P. M. (2016). Envelhecimento populacional: demandas e possibilidades na área de saúde. Séries Demográficas, 3, $153-166$.

Santos, P. H. F. D., Stival, M. M., Lima, L. R. D., Santos, W. S., Volpe, C. R. G., Rehem, T. C. M. S. B., \& Funghetto, S. S. (2020). Diagnóstico de Enfermagem de Risco de Quedas em idosos da atenção primária. Revista Brasileira de Enfermagem, 73.

Silva, J. M. S., \& Bolpato, M. B. (2017). Principais causas de quedas em idosos e atuação da enfermagem nas orientações preventivas/Main causes of falls in elderly and nursing performance in preventive guidelines/Principales causas de caídas en ancianos y actuación de la enfermeira... Journal Health NPEPS, 2(2), 418-429.

Sousa, L. M. M., Marques-Vieira, C. M. A., Caldevilla, M. N. G. N. D., Henriques, C. M. A. D., Severino, S. S. P., \& Caldeira, S. M. A. (2016). Risco de quedas em idosos residentes na comunidade: revisão sistemática da literatura. Revista Gaúcha de Enfermagem, 37(4).

Souza, L. H. R., da Silva Brandão, J. C., Fernandes, A. K. C., \& Cardoso, B. L. C. (2017). Queda em idosos e fatores de risco associados. Revista de Atenção à Saúde, 15(54), 55-60.

Tavares, R. E., Jesus, M. C. P. D., Machado, D. R., Braga, V. A. S., Tocantins, F. R., \& Merighi, M. A. B. (2017). Envelhecimento saudável na perspectiva de idosos: uma revisão integrativa. Revista brasileira de geriatria e gerontologia, 20(6), 878-889.

Teixeira, M. D. L. R. P. (2020). Efeito de uma intervenção de enfermagem de reabilitação no equilíbrio funcional e no risco de queda de idosos institucionalizados (Doctoral dissertation). 